\title{
正面光聚合实现平面到曲面的可编程形状变形“
}

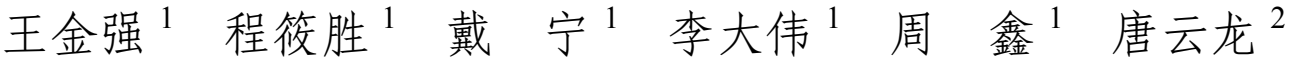 \\ (1. 南京航空航天大学机电学院 南京 210016; \\ 2. 麦吉尔大学机械工程系 蒙特利尔 $\mathrm{H} 3 \mathrm{~A} 0 \mathrm{G} 4$ 加拿大)
}

\begin{abstract}
摘要: 由平面薄片直接变形获得 3D 形状已经成为一种极具前景的设计方法, 其为生物技术、驱动器、传感器和工程中复杂 超材料的制造提供了新的手段。目前各类研究平面变形生成三维结构的方法中仍存在诸如加工材料昂贵、工艺控制繁琐和难 以独立操作的限制。考虑到正面光聚合过程中光敏树脂的聚合特性, 展示一种利用光固化过程中不匹配应力驱动平面到曲面 形状变形的设计方法。基于不同光编码模式对聚合物聚合程度的影响及沿光固化厚度方向收缩应力梯度的分布特点, 提出复 合梁理论并推导准一维梁结构的屈曲变形方程, 获得弯曲曲率与曝光剂量间的对应关系, 在此基础上, 针对准二维平面结构 变形具有分叉点和难以控制的难题, 建立弹性势能函数计算弯曲平面的能量密度, 结合有限元计算分析其变形的几何形态和 应力分布规律。最后的物理试验测试结果和花瓣, 爪子, 二十面体, 金字塔等自折叠结构的制造实例验证了方法的有效性。 关键词: 形状变形; 正面光聚合; 屈曲变形; 自折叠
\end{abstract}

中图分类号: TB124

\section{Programmable Shape-shifting from Planar Sheets to Curved Geometries via Front Photopolymerization Approach}

\author{
WANG Jinqiang $^{1} \quad$ CHENG Xiaosheng $^{1} \quad$ DAI Ning $^{1} \quad$ LI Dawei $^{1} \quad$ ZHOU Xin $^{1} \quad$ TANG Yunlong $^{2}$ \\ (1. College of Mechanical and Electrical Engineering, \\ Nanjing University of Aeronautics and Astronautics, Nanjing 210016; \\ 2. Department of Mechanical Engineering, McGill University, Montreal H3A0G4 Canada)
}

\begin{abstract}
Transforming planar sheets into desired 3D configurations has emerged as a promising design methodology, which provides new means for the fabrication of the biotechnology, actuators, sensors, and engineering of complex metamaterials. Among various approaches to producing planar-to-3D structures, there are still limitations such as high cost, tedious procedures and non-free-standing operation. Considering the polymerization characteristics of photocurable resin in front photopolymerization process, a design method for transforming planar sheets to $3 \mathrm{D}$ configurations using mismatch stress is presented. Based on the influence of discrepant photo patterns on the polymerization degree and the distribution of shrinkage stress gradient along the curing thickness, the composite beam theory is proposed theoretically. Thus, the buckling deformation equations of the quasi-one-dimensional beam are derived to obtain the relation between the bending curvature and the exposure dose. On the basis of this, for the bifurcation and rebelliousness in the quasi-two-dimensional plane structure, the elastic potential energy function is established to calculate the energy density of the bending plane, which combines with finite element calculation to reveal the deformation geometry and stress distribution law. The final mechanical experiments as well as the shape transformation examples of petal, claw, icosahedron, and pyramid verify the feasibility of our approach.
\end{abstract}

Key words: shape-shifting; front photopolymerization; buckling deformation; self-folding

\section{0 前言}

在自然界中, 生物体能利用薄片、带状结构自

* 国家自然科学基金(51775273)、江苏省自然科学基金(BK20161487)、江 苏省六大人才(GDZB-034)、江苏省重点研发计划(BE2018010-2, BE2016763)和南京航空航天大学研究生创新基金(kfjj20170523)资助项 目。20181026 收到初稿, 20190201 收到修改稿
折叠或自组装生成一些轻量化、高比强度的复杂结 构 $^{[1-2]}$ 。无论是宏观尺寸上植物的花朵 ${ }^{[3]}$ 、昆虫的翅 膀 ${ }^{[4]}$ 或微观尺寸上的细胞结构、有机分子 ${ }^{[5]}$ 都存在 这种由二维平面直接变形生成三维结构的现象。而 在实际工程应用中, 一些三维结构的制造往往伴随 着复杂且耗时费力的组装过程 ${ }^{[6-7]}$ 。利用薄片弹性 体在受到空间不均匀应力后产生的屈曲失稳变形, 
可直接将二维平面转变为特定的三维结构, 这为快 速免装配制造三维结构提供了可能 ${ }^{[8-9]}$ 。同时这种 制造方式可充分发挥功能材料响应外界刺激的特 性, 利用传统的二维加工工艺即可高效率, 低成本 地实现空间自折叠、自组装结构的制造 ${ }^{[10]}$, 在生物 医疗器械 ${ }^{[11]}$ 、药物输送装置 ${ }^{[12]}$ 、微电子系统 ${ }^{[13]}$ 、机 械超材料 ${ }^{[14]}$ 、柔性机器人 ${ }^{[15]}$ 等领域具有广阔的应 用前景。

由平面到曲面的形状变形需要基于特定的驱 动条件。一些研究学者从功能材料的刺激响应特性 出发, 研究利用水凝胶、形状记忆高分子、记忆合 金等功能材料在受到外界刺激如 $\mathrm{pH}^{[16]}$ 、温度 ${ }^{[17]}$ 、 光照 ${ }^{[18]}$ 等后发生形状变形的特点, 通过改变外界环 境驱动平面结构沿着特定方向产生伸缩、折叠或则 扭转。SYDENY 等 ${ }^{[19]}$ 将水凝胶和吸水性纤维共混 后 4D 打印出可在去离子水中自折叠生成的风车、 花瓣等三维结构; DING 等 ${ }^{[20]}$ 使用对温度具有记忆 特性的聚合物直接打印出可重复编程的三维结构。 ZHANG 等 ${ }^{[18]}$ 在聚合物薄层上印刷对光照敏感的 碳纳米管吸热收缩变形成折纸结构。这类刺激响应 变形方法的材料成本较高, 制造过程相对复杂, 变 形程度的一致性较差。而基于物理准则的变形不需 要外界环境刺激和复杂的材料合成过程, 因此可以 更高效地驱动形状变形。之前的研究证明毛细力、 剥离力、残余应力、预拉伸力等都可使二维平面产 生后屈曲变形从而实现平面到曲面的形状变形 ${ }^{[21-22]}$ 。 YAN 等 ${ }^{[23]}$ 利用后屈曲变形设计了 pop-up 结构自组 装微观的复杂三维结构; GUSEINOV 等 ${ }^{[24]}$ 利用预 拉伸的平面弹性层在去除外部约束后自由收缩形 成 CurveUps 结构。这类基于物理准则的变形方法 也存在结构设计复杂, 变形程度难以精确控制等 缺点。

正面光聚合(Front photopolymerization, FPP)可 将液态光敏树脂快速固化成含有不一致内应力的二 维薄片, 通过内应力释放可实现形状变形设计 ${ }^{[25-28]}$ 。 由于结合了光敏材料的功能特性和后屈曲变形的力 学特点, 基于 FPP 的自组装设计具有重要的研究意 义。WU 等 ${ }^{[29]}$ 发现调配光敏树脂的材料成分以及光 固化过程中的工艺参数可以控制固化聚合物收缩变 形的程度和方向。ZHAO 等 ${ }^{[25]}$ 将 FPP 与传统折纸工 艺相结合生成了金字塔、折纸鹤等折纸结构; HUANG 等 ${ }^{[26]}$ 利用 FPP 固化生成含有不同材料组分 的凝胶薄层以实现形状可变的 4D 打印; ZHANG 等 ${ }^{[23]}$ 通过调控光固化聚合物层内的残余应力进行 自组装结构的设计, 取得了较为满意的变形效果。
虽然之前的研究对 FPP 过程中聚合物的收缩变形以 及如何利用变形进行自组装设计进行了描述, 但仍 缺少材料特性、工艺参数、设计方法等对最终变形 效果影响的系统分析, 且没有提出可靠的物理模型 对变形结果进行预测和分析。

与之前的研究相比, 本文从 FPP 过程中光敏 树脂的光固化特性出发, 一方面结合试验数据分 析曝光参数和光编码结构对变形效果的影响, 建 立工艺参数与变形曲率之间的关系; 另一方面通 过建立复合梁变形方程和弹性势能函数来揭示内 应力驱动形状改变的变形机理。基于建立的数学 模型和对应的变形设计准则, 本文进行了物理试 验和有限元仿真并对形状变形结果进行分析和预 测, 从而设计最佳的工艺路线, 实现了复杂三维 结构的自组装制造。

\section{FPP 的原理与工艺特点}

\subsection{FPP 的原理}

FPP 类似于快速成型中的数字紫外光固化

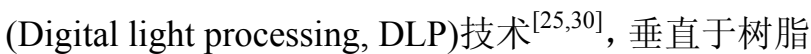
液面的投影仪投射出光掩码图像将选定区域内的光 敏树脂固化，其试验原理如图 1a 所示。与 DLP 技 术不同的是，FPP 没有逐层累加的过程，每次从一 侧连续光固化生成聚合物薄层。经过紫外光照射后, 表面的液态树脂先被固化, 随着光照时间的增加, 光敏树脂中的光聚合反应会从已固化的一侧向未固 化的一侧传递, 而光强的不断衰减导致树脂的聚合 程度逐渐降低, 使固化过程呈现梯度变化并形成不 一致的体积收缩。在如图 1a 所示紫外光照射下, 光 聚合垂直于曝光方向正向传播, 液态树脂转换为固 态聚合物, 连续的光聚合引起了顺序的体积收缩。 先固化的聚合物会限制后固化聚合物的收缩, 先固 化层受到压应力, 后固化层受到拉应力, 导致不一 致的内应力分布, 使其具有向后固化层弯曲的趋势。 在去除限制其平面外变形的玻璃板后, 聚合物薄片 释放其内应力产生向后固化层的弯曲变形。弯曲变 形的程度与 FPP 过程中的曝光强度和曝光时间相 关, 为了改变曝光强度, 采用不同灰度值(变化范围 $0 \sim 255$, 对应全黑到全白)分布的图像对曝光图案进 行光编码。利用紫外辐射计(UV-A, HANDY, Beijing) 测得不同灰度值下紫外光源的曝光强度如图 $1 \mathrm{~b}$ 所 示, 可以看出当灰度值超过 100 后, 曝光强度随灰 度值的增加几乎呈现线性关系, 说明可以通过改变 灰度值的大小来控制曝光强度。 


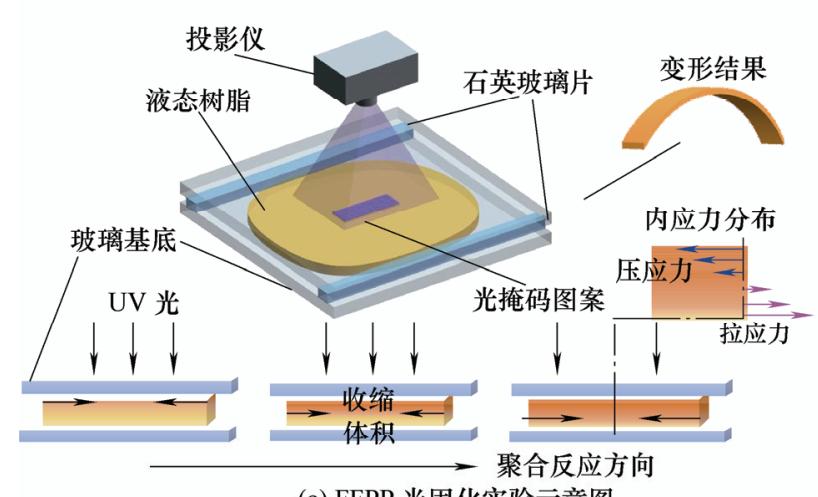

(a) FFPP 光固化实验示意图

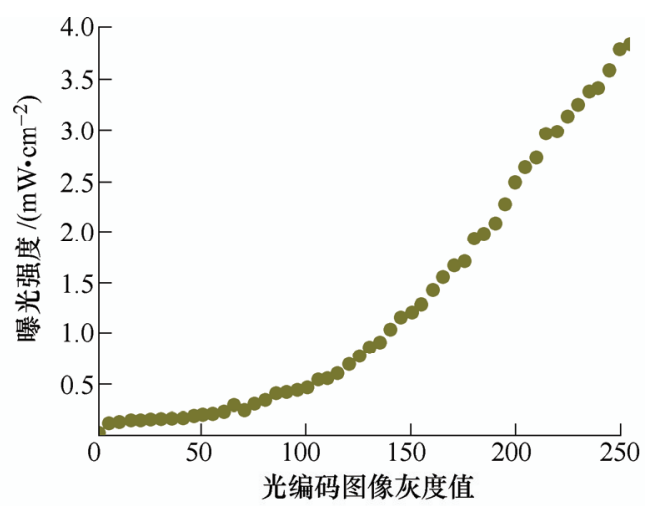

(b) 光掩码图像灰度值与曝光强度的关系

图 1 正面光聚合的试验原理图

\subsection{FPP 中体积收缩应力变形模型}

根据 SCOTT 关于无定形、共价交联聚合物网络 的理论 ${ }^{[31]}$, 在 UV 光的照射下, 液态树脂中的光引发 剂引发化学键交换反应(Bond exchange reactions, BERs), 使一端具有自由基的聚合物链与相邻的聚合 物链成键, 生成三级结构, 然后相邻键断裂将自由基 转移到新的聚合物链上, 随着化学键交替的断裂和形 成, 聚合物网络重新排列并从液态变为固态直到没有 新的自由基生成。假设紫外光沿着聚合物固化厚度方 向传播, 并规定入射表面 $z=0$ 。根据 Beer-Lambert 定律 ${ }^{[30]}$ 光在连续介质中的传播可以表述为

$$
\frac{\partial I(z, t)}{\partial z}=-A(z, t) I(z, t)
$$

式中, $I(z, t)$ 表示经过 $t$ 时间曝光后位置 $z$ 的光照强 度, $A(z, t)$ 代表 $t$ 时刻位置 $z$ 处的消光系数。曝光结 束后液态树脂中形成的聚合物交联网络为

$$
\begin{gathered}
\frac{\partial f_{O}(z, t)}{\partial t}=-k_{1} C_{R}(z, t) f_{O}(z, t)^{q} \\
f_{O}(z, t)+f_{R}(z, t)=1
\end{gathered}
$$

式中, $f_{O}$ 和 $f_{R}$ 分别代表聚合反应开始时和结束后 交联网络的体积分数; $C_{R}(z, t)$ 为树脂中自由基浓 度, $k_{1}$ 和 $q$ 为拟合系数。因此可将聚合物内部的内 应力表示为 ${ }^{[28]}$

$$
\begin{gathered}
\sigma(z, t)=E\left(1-f_{R}(z, t)\right)\left(\varepsilon_{s}^{0}+\Delta \varepsilon(z, 0, t)\right)+ \\
E \int_{0}^{t} \frac{\partial f_{R}(z, \tau)}{\partial t} \Delta \varepsilon(z, \tau, t) \mathrm{d} \tau
\end{gathered}
$$

式中, $E$ 为聚合物的弹性模量; $\varepsilon_{s}^{0}$ 为最初的收缩应 变; $\Delta \varepsilon(z, 0, t)$ 代表从体积收缩开始 $(t=0)$ 到曝光结 束后聚合物的体积收缩增量; $\Delta \varepsilon(z, \tau, t)$ 代表从时间 $\tau$ 到时间 $t$ 的收缩应变增量。利用表 1 中的参数可获 得 FPP 中固化聚合物的内应力, 但计算过程复杂繁 琐, 因此这里提出基于 FPP 过程中不一致体积收缩 来计算其内应力的方法。
FPP 是一个空间上的动态过程, 聚合物的聚合 程度与曝光时间 $(t)$ 和聚合反应发生的位置 $(z)$ 相 关。这里我们引入一个动态的有序参数 $\varphi(z, t)$ 来描 述 FPP 过程中的聚合程度 ${ }^{[33]}(0 \leqslant \varphi \leqslant 1$, 代表树脂由 液态变为完全的固态), 它正比于光照强度 $I(z, t)$ 与 可参加聚合反应的液态树脂 $(1-\varphi(z, t))$ 的乘积

$$
\frac{\partial \varphi(z, t)}{\partial t}=K[1-\varphi(z, t)] I(z, t)
$$

式中， $K$ 为反应常数，结合方程式(1)和(5), 可获得 关于 $\varphi$ 的解析方程

$$
\varphi(z, t)=1-\exp \left[-K I_{0} \exp \left(-A_{0} z\right) t\right]
$$

式中， $I_{0}$ 代表表面处 $(z \equiv 0)$ 的光照强度; $A_{0}$ 代表有 效的消光系数, 一般默认其为常数 ${ }^{[30]}$ 。随着光聚合 的进行，聚合物的交联密度逐渐增加到超过转换阈 值 $\varphi_{c}$ 时, 树脂由液态转化为固态, 随着曝光时间的 增加, 固态树脂逐渐累积形成一定的固化厚度

$$
\begin{aligned}
z\left(\varphi=\varphi_{c}\right)=z_{f}(t) \\
z_{f}=\frac{\ln \left[K I_{0} t / \ln \left(1 /\left(1-\varphi_{c}\right)\right)\right]}{A_{0}}
\end{aligned}
$$

式(7)说明 FPP 中的固化厚度 $z_{f}$ 随着曝光时间 $t$ 和初 始曝光强度 $I_{0}$ 增加而增加, 因此可以通过改变曝光 时间和曝光图像的灰度值控制固化厚度。如图 1a 所示, 在 UV 光下一定固化厚度的聚合物薄层会很 快形成, 后续的光照使聚合物的交联程度逐渐沿着 固化厚度方向增加, 形成沿着固化厚度方向不一致 的聚合程度(颜色越深，固化程度越高)。随着聚合 物交联程度的增加, 聚合物的弹性模量也会增加, 其与聚合程度 $\varphi$ 之间满足 ${ }^{[29]}$

$$
E(\varphi)=E_{c} \exp \left(b\left(\varphi-\varphi_{c}\right)\right)+E_{d}
$$

式中, $E_{c}, E_{d}, b$ 都是拟合参数。树脂由液态转化为 固态时, 体积发生收缩, 这里引入 Hencky 应变 ${ }^{[33]}$ 将整个过程的收缩应变转化为一个与 $\varphi$ 相关的函 
数, 将 FPP 过程中的内应力表示为

$$
\sigma=E \varepsilon^{s}=E(\varphi)\left(\varepsilon-\varepsilon^{s}(\varphi)\right)
$$

式中, $\varepsilon^{s}$ 为收缩应变, 因此不同位置处的聚合程度 $\varphi$ 差异越大, 聚合物内部的应力差越大, 更容易引 起沿着某个方向的变形。由于 FPP 过程中限制了已 固化聚合物在平面外的运动, 只存在平面内的拉伸, 已固化的聚合物满足力学平衡条件即

$$
\int_{0}^{z_{f}} \sigma \mathrm{d} z=\int_{0}^{z_{f}} E\left(\varepsilon-\varepsilon^{s}\right) \mathrm{d} z=0
$$

式中, $\varepsilon$ 为总应变, 且满足 $\varepsilon=\varepsilon^{s}+\varepsilon^{e}, \varepsilon^{e}$ 代表弹 性应变, $E$ 和 $\varepsilon^{s}$ 都是与 $\varphi$ 有关的函数, 且当聚合物 中交联程度 $\varphi<\varphi_{c}$ 时, 应变以及弹性模量均为零。 去除对聚合物层的约束后其会释放内应力并自由变 形, 但仍然满足力和力矩的平衡 ${ }^{[34]}$, 即

$$
\begin{gathered}
\int_{0}^{z_{f}} \sigma \mathrm{d} z=\int_{0}^{z_{f}} E \varepsilon^{e} \mathrm{~d} z=0 \\
\int_{0}^{z_{f}} z \sigma \mathrm{d} z=\int_{0}^{z_{f}} z E \varepsilon^{e} \mathrm{~d} z=0
\end{gathered}
$$

通常将这类薄层结构看作非欧几里得平面(nonEuclidean plates, NEP) ${ }^{[35]}$, 其平衡状态是由拉伸势能 $E_{s}$ 和弯曲势能 $E_{b}$ 两种能量在薄层内相互竞争共同 决定的, 弹性势能驱动薄层弯曲, 拉伸势能驱动薄 层伸展。ZHAO 等提出将弯曲和拉伸叠加, 获得聚 合物薄层的弹性应变 ${ }^{[27]}$

$$
\varepsilon^{e}=\varepsilon-\varepsilon^{s}+\varepsilon^{b}+\kappa\left(z-z_{f} / 2\right)
$$

这里的弯曲应变 $\varepsilon^{b}$ 是通过测量薄层中间平面 $z=z_{f} / 2$ 处的弯曲曲率 $\kappa$ 获得的, 但在实际的 FPP 中, 如图 1a 所示, 聚合程度 $\varphi$ 并不是线性变化, 中 性面并不总是在 $z=z_{f} / 2$ 处。此外我们发现聚合物 薄层的几何形态对弯曲曲率 $\boldsymbol{\kappa}$ 有很大影响, 式(13) 的计算结果会产生较大误差, 因此本文将聚合物薄 层分为准一维梁结构和准二维圆盘结构分别研究其 变形过程, 结合其变形特点选择合理工艺参数, 建

表 1 理论模型中使用的参数及其出处

\begin{tabular}{lcl}
\hline \multicolumn{1}{c}{ 参数 } & 数值 & 出处 \\
\hline 气体常数 $R /(\mathrm{J} /(\mathrm{K} \cdot \mathrm{mol}))$ & 8.314 & 文献[30] \\
光引发剂初始浓度 $C_{I}(0) /(\mathrm{mol} / \mathrm{L})$ & 0.052 & 计算值 \\
相位演化因子 $k_{1}$ & 2.0 & 拟合值 \\
相位演化指数 $q$ & 2.0 & 拟合值 \\
光聚合反应常数 $K /\left(\mathrm{cm}^{2} \cdot \mathrm{mW}^{-1} \cdot \mathrm{s}^{-1}\right)$ & 0.03 & 文献[25] \\
弹性模量匹配系数 $E_{c} / \mathrm{MPa}$ & 0.19 & 拟合值 \\
拟合系数 $E_{d} / \mathrm{MPa}$ & 0.32 & 拟合值 \\
误差系数 $b$ & 5.56 & 拟合值 \\
转化阈值 $\varphi_{c}$ & 0.2 & 计算值 \\
消光系数 $A_{0} / \mathrm{mm}^{-1}$ & 5 & 文献[27] \\
\hline
\end{tabular}

立变形形态与参数间的定量关系, 从而对变形结果 进行合理预测和控制。

\section{2 试验流程}

\section{1 试验材料}

本文选取相对分子量为 700 的聚乙二醇二丙烯 酸酯(PEGDA)作为聚合单体，苯基(2,4,6-三甲基苯 甲酰基)双氧化膦(Irgacure 819)作为光引发剂, Sudan $\mathrm{I}$ 作为光吸收剂, 聚乙二醇(PEG)作为分散剂。最终 获得 FPP 过程中的光敏树脂各组分的质量占比为: 90.23\% PEGDA、9\% PEG、0.66\% Irgacure 819 和 $0.11 \%$ Sudan I。试验材料从 Sigma-Aldrich 和上海 Macklin 公司购买。树脂的制备要避开紫外光源, 恒温脱气后静置待用。

\section{2 聚合物性能测试}

$\mathrm{FPP}$ 过程中 $\mathrm{C}=\mathrm{C}$ 的转换率可以表征液固转化程 度 ${ }^{[29]}$, 将聚合反应中不变的 $\mathrm{C}=\mathrm{O}$ 双键的吸收峰 $A_{1725}$ 作为标准化因子, 则转换程度 $p$ 为

$$
p=1-\frac{\left(A_{t}^{1620}+A_{t}^{1635}\right) / A_{t}^{1725}}{\left(A_{0}^{1620}+A_{0}^{1635}\right) / A_{0}^{1725}}
$$

式中, $A_{t}$ 代表照射时间 $t$ 后吸收峰, $A_{0}$ 代表未经紫外 照射的吸收峰, 将 $\mathrm{C}=\mathrm{C}$ 的转换程度进行正则化得到 FPP 中的聚合程度 $\varphi=p / p_{\text {max }}$ 。利用红外光谱仪 (NEXUS670,Thermo Nicolet, USA)测量不同固化条 件下聚合物的吸收峰如图 2a 所示, 液态光敏树脂在 不同光掩码下曝光 $10 \mathrm{~s}$ 后, 随着曝光剂量 $D$ (曝光 强度 $\times$ 曝光强度)的增加, $A_{t}$ 和 $A_{0}$ 均会下降, 说明聚 合程度在逐渐增加, 树脂由液态逐渐转化为固态。 为了确定光敏树脂的转换阈值 $\varphi_{c}$, 分别测量经过不 同灰度值的矩形光掩码 $(50 \mathrm{~mm} \times 4 \mathrm{~mm})$ 曝光后,沿固 化厚度方向各处的 $\mathrm{C}=\mathrm{C}$ 吸收峰, 如图 $2 \mathrm{~b}$ 所示, 虽然 随曝光时间 $t$ 的增加, 树脂的聚合程度 $\varphi$ 增加, 但只 有 $\varphi_{c}>0.2$ 时液态树脂才会固化。结合式(7)获得不同 曝光参数的聚合物的固化厚度如图 $2 \mathrm{c}$ 所示, 随曝光 时间 $t$ 增加, 固化厚度 $z_{f}$ 逐渐增加且同一时刻的曝光 强度 $I_{0}$ 越大, 固化厚度也越大, 这与利用 ImageJ 图 像处理插件 ${ }^{[36]}$ 处理后获得的实际固化聚合物的 $z_{f}$ 具 有较好的一致性。直接测量光固化过程中光敏树脂 的体积收缩率比较困难, 但 FPP 过程中的体积收缩 与 $\varphi$ 呈线性关系 ${ }^{[25]}$, 即 $\Lambda=\chi \varphi$, 其中 $\chi$ 是与材料自 身有关的拟合系数。当拟合系数 $\chi=21.23$ 时, 发现 聚合物的体积收缩率与聚合程度为如图 $2 \mathrm{~d}$ 所示的正 比例关系，且最大的体积收缩率为 $18 \%$ 。 


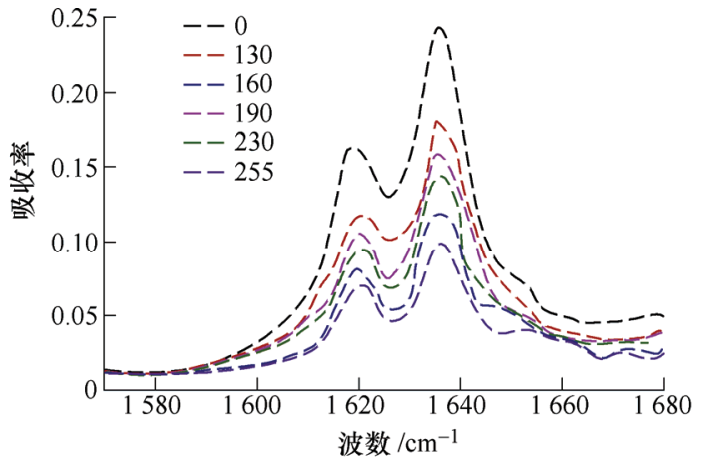

(a) 经过光掩码曝光后树脂的红外光谱吸收峰

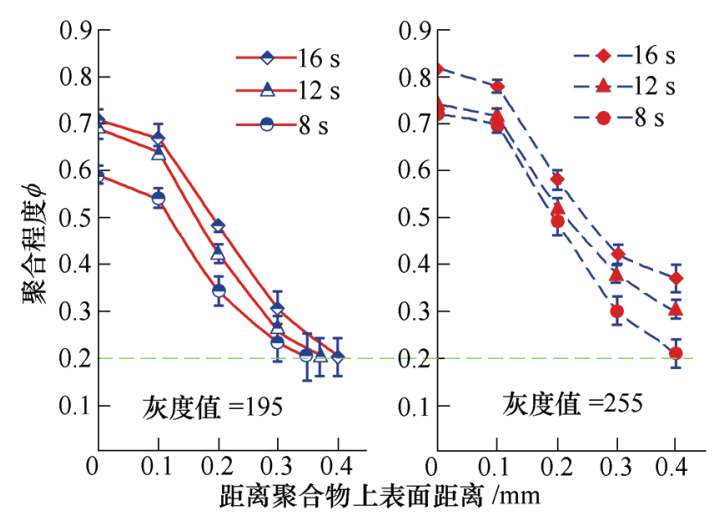

(b) 沿固化厚度方向不同位置处的聚合程度

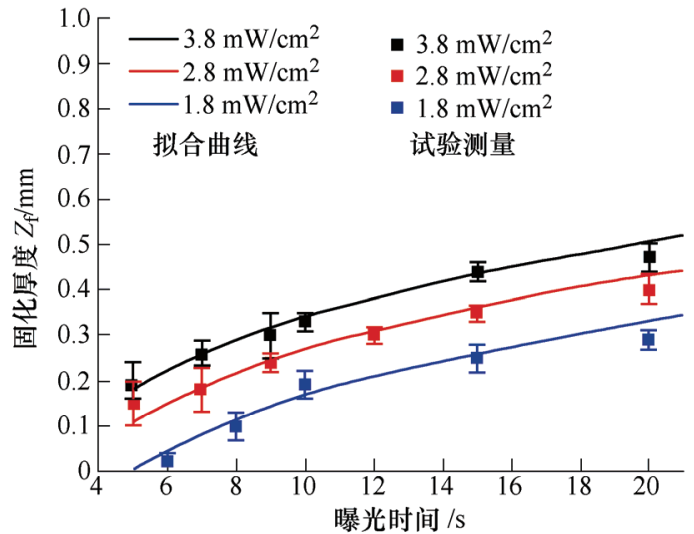

(c) 固化厚度随曝光时间的变化

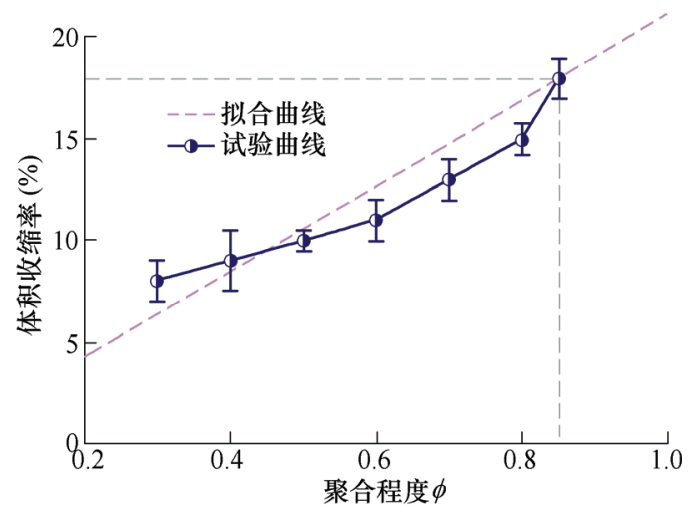

(d) 不同聚合程度下的体积收缩率

图 2 光聚合反应中的材料性能变化

\section{3 聚合物固化后内应力计算}

得到转换阈值 $\varphi_{c}$ 后, 据式(8)获得不同固化程度 下聚合物的弹性模量 $E_{\varphi}$, 计算结果与利用单轴力学 测试仪(WDW-50, Shanghai)试验测试结果如图 3a 所
示, 随聚合程度 $\varphi$ 增加, 弹性模量 $E_{\varphi}$ 近似呈现指数 增加, 固化聚合物的聚合程度越大其弹性模量也越 大。结合图 $2 \mathrm{c}$ 中的转化阈值和式(13), 获得图 $3 \mathrm{~b}$ 所示不同曝光强度下距离固化上表面 $z_{d}$ 处的应力 $\sigma$ 。先固化的聚合物受到压应力而后固化的聚合物 受到拉应力, 随着固化厚度 $z_{f}$ 的增加, 树脂的聚合 程度 $\varphi$ 降低, 其内部的应力也降低。利用图 $3 b$ 的计 算结果和式(7)、(10) (13)获得如图 3c 所示曝光时 间与聚合物变形程度间的关系，不同曝光强度 $I$ 下 固化聚合物弯曲半径与曝光时间 $t$ 的变化曲线, 随 着曝光时间的增加, 树脂的聚合程度和弹性模量增 加, 弯曲刚度也会增加, 因此聚合物的弯曲变形逐 渐减少。式(13)中 ZHAO 直接取聚合物薄层的中性

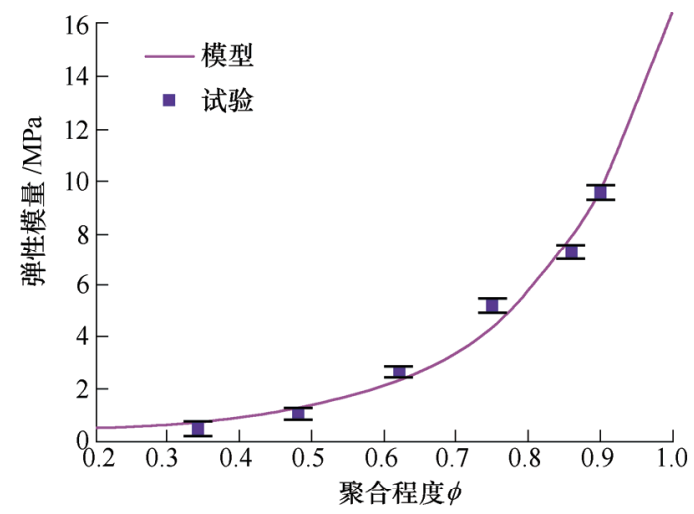

(a) 聚合物弹性模量与聚合程度间的变化

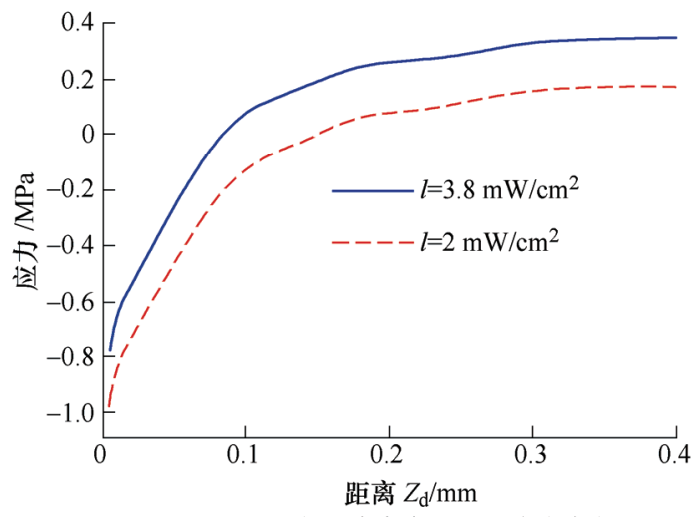

(b) 沿固化厚度方向各处的应力变化

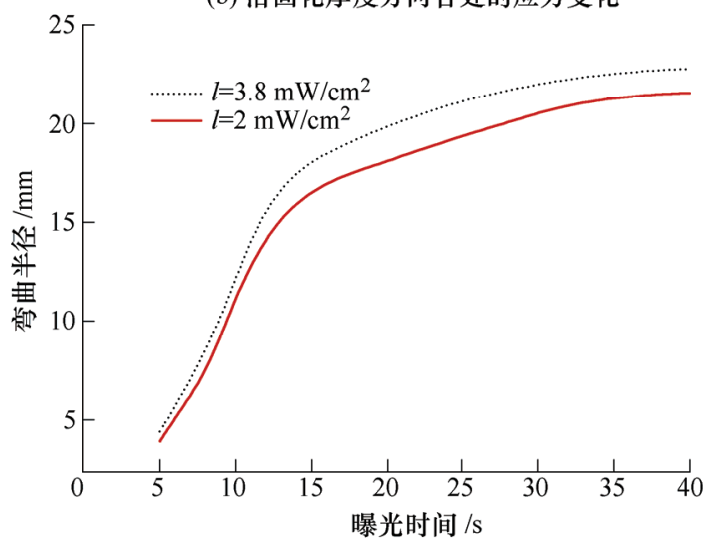

(c) 聚合物弯曲半径随曝光时间的变化

图 3 聚合物固化后的性能曲线 
面为 $z=z_{f} / 2$, 但图 $1 \mathrm{a}$ 说明沿固厚度化方向各处的 聚合程度并不是线性变化, 因此图 3c 并不能完全 说明 FPP 的变形曲率的分布特点。为了说明几何形 态也对变形结果有影响, 利用矩形 $(50 \mathrm{~mm} \times 4 \mathrm{~mm})$ 和圆形 $(r=35 \mathrm{~mm})$ 光掩码固化了一系列准一维的 梁结构和准二维的圆盘结构, 并利用三维扫描仪 (3 Shape Q-750, Denmark)获取固化后聚合物的弯曲 曲率。

\section{3 内应力驱动平面到曲面变形}

\section{1 准一维梁结构的变形}

FPP 过程中聚合物的厚度很薄, 当薄层的长度 是厚度的 60 倍以上, 且宽度也远小于长度时, 可将 其将其看作是准一维的梁结构 ${ }^{[37]}$ 。因为沿固化厚度 方向各处聚合程度不同, 所以这里建立基于 EulerBernoulli 方程的复合梁模型来求解树脂固化后弯曲 的曲率。由于复合梁结构的截面尺寸较小, 其弯曲 主要由沿固化厚度方向的体积收缩引起, 弯曲半径 满足

$$
\kappa=\frac{1}{\rho}=\frac{\int_{0}^{z_{f}} E \varepsilon^{s}(z-\tilde{z}) \mathrm{d} z}{\int_{0}^{z_{f}} E(z-\tilde{z})^{2} \mathrm{~d} z}
$$

式中, $\rho$ 为弯曲半径; $\tilde{z}$ 为准一维梁结构的中性轴 位置, 可表示为

$$
\tilde{z}=\frac{\int_{0}^{z_{f}} E z \mathrm{~d} z}{\int_{0}^{z_{f}} E \mathrm{~d} z}
$$

式(15)中的分子项为聚合物的弯曲力矩, 分母项为 弯曲刚度。图 4 是当曝光强度为 $3.8 \mathrm{~mW} \cdot \mathrm{cm}^{-2}$ 和 $2 \mathrm{~mW} \cdot \mathrm{cm}^{-2}$ 时, 利用矩形光掩码曝光不同时间后获 得固化聚合物的弯曲半径, 当光照射剂量 $D$ (曝光强 度 $\times$ 曝光时间 $)<15 \mathrm{~mJ} \cdot \mathrm{cm}^{-2}$ 时, 树脂表面接收到的 紫外光辐照能量不足以使其充分固化, 聚合物的弹 性模量 $E$ 和收缩应变 $\varepsilon^{s}$ 都较小, 其内部的应力不足 以克服弯曲刚度而产生较大变形; 当 $D>50 \mathrm{~mJ} \cdot \mathrm{cm}^{-2}$ 时, 聚合物上表面接收足够的光照后具有较大的 $E$ 和 $\varepsilon^{s}$, 但由于光的散射和衰减, 下表面的聚合程度 仍然较低, 上下表面模量和应变差使复合梁产生明 显弯曲变形; 随曝光剂量 $D$ 继续增加, 下表面的树 脂继续固化, 但已完全固化的树脂会阻碍紫外光的 传播并加速光的衰减, 因此下表面聚合物的 $E$ 和 $\varepsilon^{s}$ 缓慢增加; 当 $D>50 \mathrm{~mJ} \cdot \mathrm{cm}^{-2}$ 时, 聚合物内部的不 一致性逐渐减小, 弯曲刚度的增加速率超过了弯曲 力矩, 此时聚合物弯曲变形程度逐渐减小。最终树
脂完全固化, 弯曲力矩趋于 0 , 聚合物不再发生弯 曲变形。图 4 右上角插图是利用式(15)拟合的理论 弯曲曲率变化曲线, 与图 3c 相比, 图 4 更符合试验 结果的变化趋势, 说明复合梁理论能更好的表征 FPP 中准一维梁结构的变形, 也说明可以通过改变 曝光剂量 $D$ 来控制聚合物的变形曲率 $\kappa$ 。

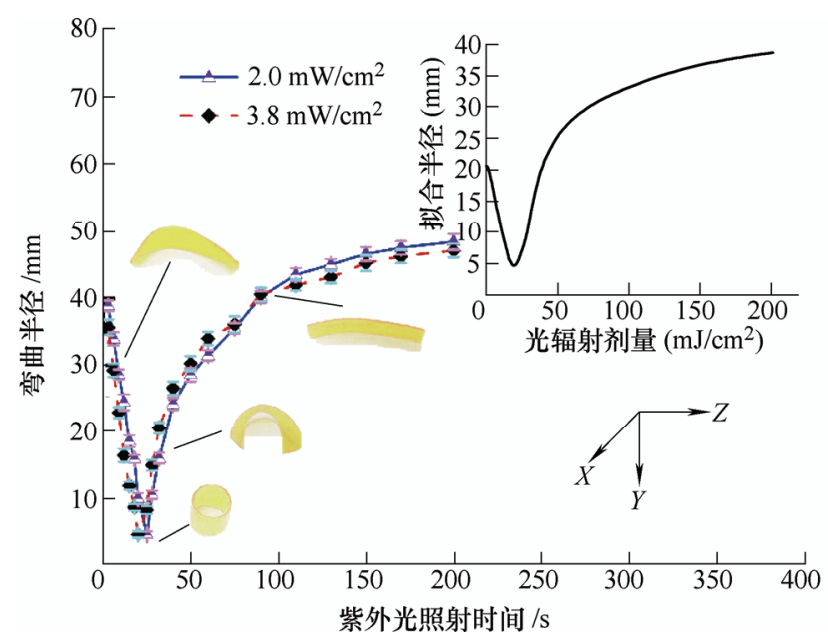

图 4 梁结构弯曲半径随曝光时间的变化

\section{2 准二维圆盘结构的变形}

当 FPP 中固化聚合物的长度和宽度差别不大 时, 可视其为准二维的结构, 这里以圆盘结构 $(r=35$ $\mathrm{mm}$ )为例分析变形的规律。如图 5a 所示, 随着曝光 剂量的增加, 圆盘发生屈曲变形, 将测量得到的通 过圆心互相垂直的曲率 $\kappa_{1}, \kappa_{2}$ 正则化后得到弯曲曲 率随曝光剂量的变化曲线(图 5b)。发现准二维结构 的变形中存在曲率分叉点。由 NEP 能量方程 ${ }^{[33]}$ 知, 准二维平面的屈曲变形是由拉伸 $E_{s}$ 和 $E_{b}$ 弯曲共同 作用。开始时聚合物的厚度较薄, 弯曲应力 $E_{b}$ 占主 导地位使其轴对称弯曲 $\left(\kappa_{1}=\kappa_{2}\right)$; 随曝光剂量 $D$ 增 加, 固化厚度增加引起拉伸应力 $E_{s}$ 增加, $E_{s}$ 和 $E_{b}$ 相 互竞争造成平面屈曲失稳变形 $\left(\kappa_{1} \neq \kappa_{2}\right)$; 随固化厚 度的继续增加，拉伸应力 $E_{\mathrm{s}}$ 占主导地位使薄片向四 周对称拉伸 $\left(\kappa_{1} \approx \kappa_{2}\right)$, 但不会恢复到平整状态。为了 求解准二维平面结构的变形曲率, 首先简化 FPP 过 程中的应力梯度, 将其看作双层圆盘 $(R)$, 其中上 部分弹性模量为 $E_{t}$, 厚度为 $h_{t}$, 泊松比为 $v_{t}$, 对应 下半部分为 $E_{b}, h_{b}$ 和 $v_{b}$, 然后建立关于圆盘的极 坐标 $\left(E=\left(E_{b}+E_{t}\right) / 2, h_{f}=h_{b}+h_{t}, v=\left(v_{b}+v_{t}\right) / 2\right)$, 将内应力表示为 $\left(\sigma_{r r}(r, z), \sigma_{\theta \theta}(r, z)\right)$, 因此则系统的 总势能 ${ }^{[38]}$ 可用应变 $\varepsilon(r, z)$ 表示为

$$
V=2 \pi \int_{0}^{R} \int_{-h_{b} / 2}^{h_{f}+h_{b} / 2} \frac{E\left(\varepsilon_{r r}^{2}+\varepsilon_{\theta \theta}^{2}+2 v \varepsilon_{r r} \varepsilon_{\theta \theta}\right) r}{2\left(1-v^{2}\right)} \mathrm{d} r
$$

由于结构的对称性, 采用 Kirchhoff 假设 ${ }^{[39]}$, 即 垂直于基板中截面的平面在变形中保持垂直于中截 
面, 将中截面的应变表示为径向应变 $u_{r}(r)$ 和轴向应 变 $u_{z}(r)$ 。根据 von Karman ${ }^{[35]}$ 弹性理论, 如果 $u_{r}=\varepsilon_{0} r+\varepsilon_{1} r^{3}, u_{z}=\kappa r^{2} / 2$, 且 $\frac{\partial V}{\partial \varepsilon_{0}}=0, \frac{\partial V}{\partial \varepsilon_{1}}=0$,

可将圆盘内部的不匹配应力 $S$ 表示为曲率 $\kappa$ 的函数

$$
\begin{gathered}
S=K\left[1+\left(1-v_{b}\right) K^{2}\right] \\
K=R^{2} \kappa / 4 h_{b} \\
S=3 \varepsilon_{m} R^{2} h_{t} E_{t}\left(1-v_{b}\right) / 2 h_{b}^{3} E_{b}\left(1-v_{t}\right)
\end{gathered}
$$

此时圆盘有唯一的曲率 $\kappa$, 但实际过程中 $h_{b} \approx h_{f}$ 很 难保证, 所以式(17)并不总是成立。此时考虑 $u_{z}(x, y)=\left(\kappa_{x} x^{2}+\kappa_{y} y^{2}\right) / 2$, 将平面的内部应变 $\varepsilon_{x x}, \varepsilon_{y y}, \varepsilon_{x y}$ 用 $\kappa_{x}, \kappa_{y}$ 和等另外 6 个量纲一参数表示, 系统总势能为 $V\left(\kappa_{x}, \kappa_{y}, a_{1}, \cdots, b_{3}\right)$, 但直接求解仍很 困难。这里取一个面积为 $A=\pi R^{2}$ 的圆盘, $u, v, w$ 是 笛卡尔坐标系下圆盘沿 $x, y, z$ 方向的位移。然后利 用有限元法将圆盘网格化, 赋予节点材料属性和单 元型函数, 外部载荷使节点向能量最低的位置运动, 并计算节点的应力 $(\sigma)$ 应变 $(\varepsilon)$ 关系, 获得单元的应 力能密度 $U_{e l}^{d}=\varepsilon^{T} \sigma / 2$, 这样就可以利用有限元软件 模拟出二维平面的变形过程, 并其将总势能方程拆 分为弯曲势能 $U_{b}$ 和拉伸势能 $U_{s}{ }^{[40]}$

$$
\begin{gathered}
U_{b}=\frac{1}{2} \iint_{A} \frac{E z_{f}^{3}}{12\left(1-v^{2}\right)}\left[\left(\frac{\delta^{2} \omega}{\delta x^{2}}\right)^{2}+\left(\frac{\delta^{2} \omega}{\delta y^{2}}\right)^{2}+2 v \frac{\delta^{2} \omega}{\delta x^{2}} \frac{\delta^{2} \omega}{\delta y^{2}}+2(1-v)\left(\frac{\delta^{2} \omega}{\delta x \delta y}\right)^{2}\right] \mathrm{d} x \mathrm{~d} y \\
U_{s}=\frac{1}{2} \iint_{A} \frac{E z_{f}}{\left(1-v^{2}\right)}\left[\left(\frac{\delta u}{\delta x}+\frac{1}{2}\left(\frac{\delta \omega}{\delta x}\right)^{2}\right)^{2}+2 v\left(\frac{\delta u}{\delta x}+\frac{1}{2}\left(\frac{\delta w}{\delta x}\right)^{2}\left(\frac{\delta v}{\delta y}\right)^{2}\right)\right] \\
\left.+\frac{1}{2}(1-v)\left(\frac{\delta u}{\delta y}+\frac{\delta v}{\delta x}+\frac{\delta \omega}{\delta x} \frac{\delta \omega}{\delta y}\right)^{2}+\left(\frac{\delta v}{\delta y}+\frac{1}{2}\left(\frac{\delta \omega}{\delta y}\right)^{2}\right)^{2}\right] \mathrm{d} x \mathrm{~d} y
\end{gathered}
$$

借鉴之前对双层聚合物屈曲变形的有限元仿真 研究 $^{[40-42]}$ 可利用热应力代替收缩应力。将薄层看作 是由正、负两种不同膨胀系数材料组成的薄壳, 选 取复合壳截面, 应用中轴网格划分算法划分四边形 网格单元, 固定质心节点并限制其刚体的位移, 最 后设置温度梯度, 在热应力下驱动结构变形。为了 验证方法的可行性, 首先在 ABAQUS (Dassault Systems, Waltham, MA,USA)中建立一个尺寸为 50 $\mathrm{mm} \times 4 \mathrm{~mm} \times 0.4 \mathrm{~mm}$ 的梁模型并考虑对称边界条件,
固定顶部表面节点的移动, 利用 S4R 单元进行网格 划分 ${ }^{[38]}$, 选取材料的弹性模量为 $8 \mathrm{MPa}$, 泊松比为 0.25 。沿厚度方向分别赋予材料热膨胀系数为 $f(z)=2-20 z$ 和 $f(z)=3.8-20 z$ 模拟曝光强度为 2 和 $3.8 \mathrm{mWcm}^{-2}$ 时的弯曲变形。图 $5 \mathrm{c}$ 显示仿真结果 沿厚度方向存在与第 1.3 节 FPP 固化过程中类似的 应力梯度, 且增大材料的热膨胀系数可以增加弯曲 程度, 这与增加曝光剂量对变形的影响相同, 说明 可以利用温度场来模拟 FPP 中的弯曲变形, 且可通

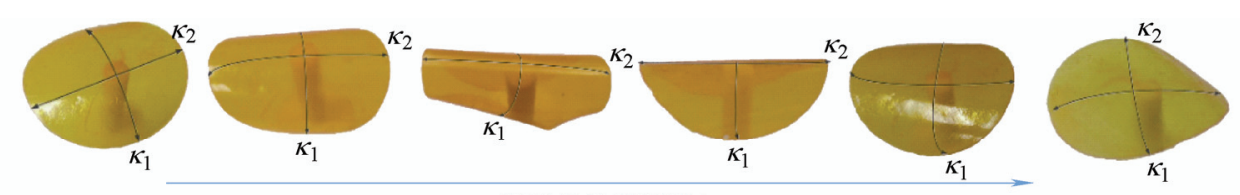

曝光剂量 逐渐增加

(a) 不同曝光剂量下圆㭧结构的曲率变化

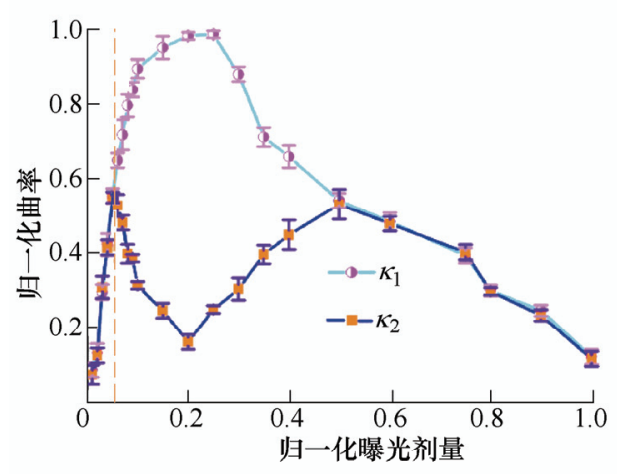

(b) 归一化光照剂量下圆盘结构的曲率

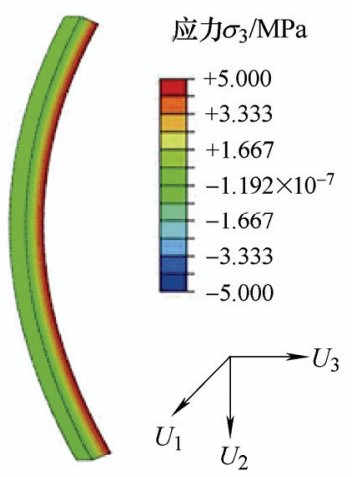

$F(z)=20-3.8 z$

(c) 不同热胀膨系数下梁结构的弯曲模拟 


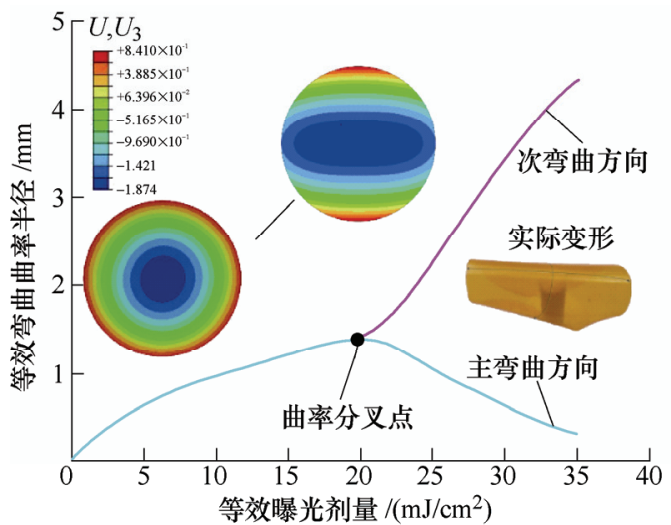

(d) 圆形平面屈曲变形模拟

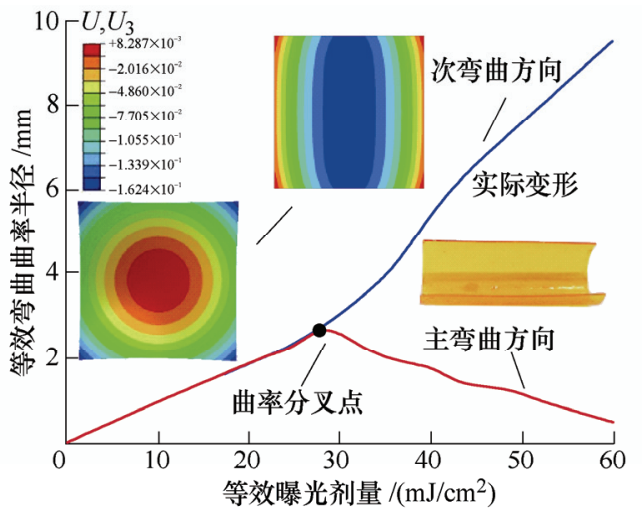

(e) 矩形平面屈曲变形模拟

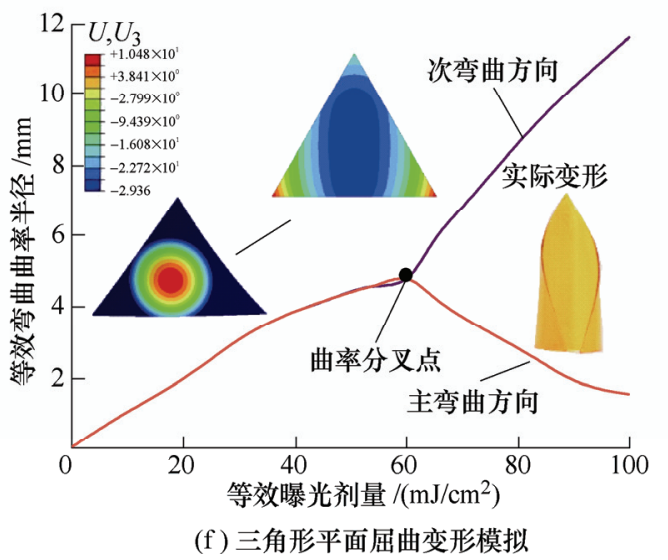

图 5 准二维结构的弯曲变形及其仿真模拟

过改变热膨胀系数控制变形程度。为了说明准二维 平面的变形特点, 分别对圆 $(r=35 \mathrm{~mm})$ 、矩形 $(50$ $\mathrm{mm} \times 4 \mathrm{~mm})$ 和等边三角形平面 $(a=40 \mathrm{~mm})$ 进行模拟 仿真。利用式(18)和(19)将弹性势能最小化得到 $U_{3}$ 方向的应力应变, 根据应变可得到对应的弯曲曲率, 同时将不匹配应力看成等效曝光剂量, 得到模拟结 果如图 5d 5f, 随等效曝光剂量的增加, 准二维结 构的曲率出现了分叉点, 在分差点之前两个曲率基 本相同, 准二维结构对称弯曲, 在分差点之后, 主 曲率和次曲率同时存在, 二维平面发生屈曲变形, 且随曝光剂量增加主、次曲率间的差值增大, 屈曲 变形更明显, 说明准二维结构的变形同样可以通过 曝光剂量 $D$ 来控制。

\section{4 基于 FPP 的平面到曲面形状变形}

在第 1 节中分析了 FPP 中收缩应力产生的原因 和特点, 第 2 节利用试验证明了 FPP 中应力梯度的 存在并研究了不同曝光参数对变形程度的影响, 第 三节利用是复合梁和势能函数分别分析了曝光剂量 $D$ 对准一维梁结构和准二维圆盘结构变形曲率间 的关系, 结合有限元证明了可以通过调节曝光剂量 调节收缩应变的大小, 改变不一致应力的分布, 从
而实现平面到曲面的形状变形。根据以上的物理模 型和试验方法设计了如图 6 所示的基于 FPP 的平面 到曲面形状变形的工艺路线。首先提取原始模型(马 蹄莲花)表面形状的曲率信息(图 6a), 根据图 $6 \mathrm{~b}$ 的 函数关系, 选择对应的曝光剂量 $D$, 在确定曝光时 间 $t$ 为 $10 \mathrm{~s}$ 后可获得对应的曝光强度 $I$, 利用图 $1 \mathrm{~b}$ 中所示的曝光强度与灰度值的关系确定各处的灰度 值(图 6c), 照此获得最终的光编码图像(图 6d), 最 后利用 FPP 生成需要的自变形结构(图 6e)。通过图 6 的工艺路线, 可以将平面卷曲成圆柱(图 7a), 随 着平面内弯曲和拉伸的竞争, 平面的不稳定性增加 则生成雉面结构(图 7b)。利用 FPP 自折叠生成的抓 手(图 7c), 经过二次曝光固化可以抓取其自身重量 25 倍的重物。利用交联程度低的聚合物具有更大的 体积收缩, 可将其作为连接平面的铰链, 驱动平面 结构自组装生成图 $7 \mathrm{~d}$ 中的金字塔(平板 $g=250$, 连 接处 $g=140, t=10 \mathrm{~s}$ ) 和正二十面体(平板处 $g=255$, 连接处 $g=150, t=9 \mathrm{~s}$ )。平面内的收缩应力梯度的 变化增加了梁结构的不稳定性可引起卷曲变形, 利 用灰度值交错变化的光掩码图像可生成花瓣和花荵 结构(图 7e)。试验结果说明本文的方法能迅速准确 地将三维模型中的各处的曲率 $\left(\kappa_{A}, \kappa_{B}, \kappa_{C}, \kappa_{D}\right)$ 转换 为对应的光编码图像的灰度值 $\left(g_{A}, g_{B}, g_{C}, g_{D}\right)$, 从而 
对 FPP 过程进行参数化设计, 提供更合理的工艺路 用更简洁的试验控制参数和工艺材料实现了平面到 线, 因此与 ZHAO 和 HUANG 的方法相比, 本文利 曲面的形状变形，且提升了制造的效率和准确性。

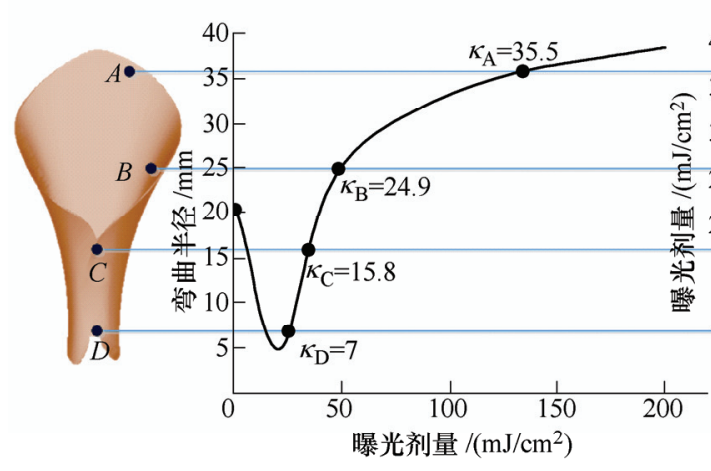

(a) 原始三维 模型 (b) 曝光剂量与弯曲半径的关系

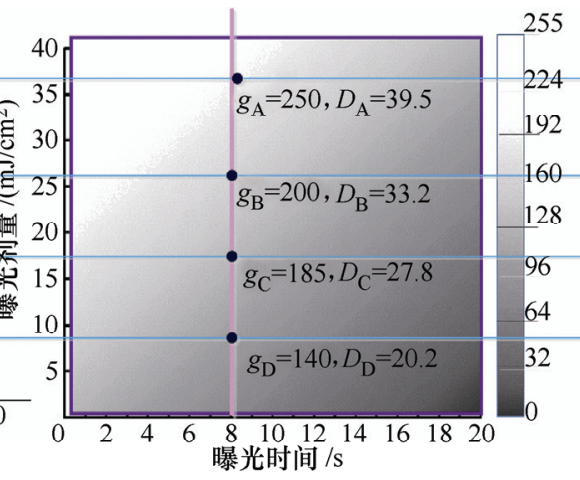

(c) 光掩码灰度值随曝光时间的分布

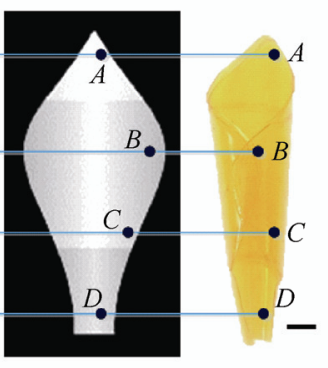
(d) 光编码灰度 (e)FPP 固化生成

图 6 自变形结构设计路线图

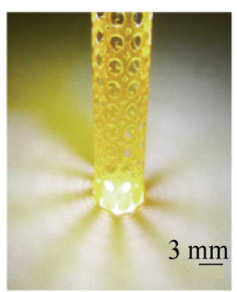

(a) 圆筒结构

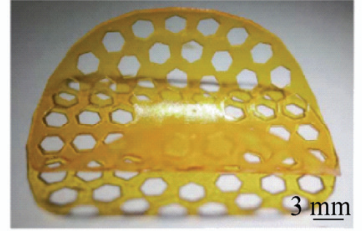

(b) 锥面结构

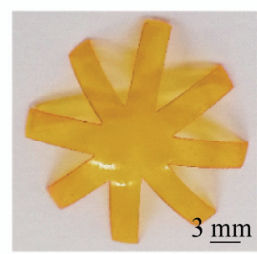

(c) 自折叠抓手

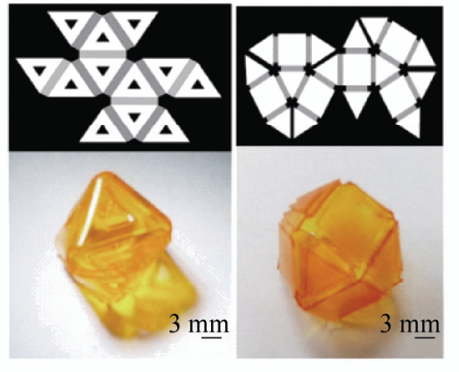

(d) 自组装金字塔和二十面体

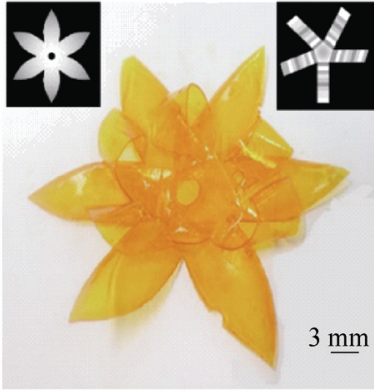

(e) 自组装生成仿花瓣结构

图 7 利用 FPP 生成的自变形结构

\section{5 结论}

(1) 提出了一种通过设计正面光聚合中光编 码投影图像的灰度值和曝光时间来将二维平面迅速 地转化为特定三维结构的工艺方法, 然后在对固化 聚合物材料性能参数测试的基础上结合建立的力学 模型和有限元仿真方法, 分析了 FPP 过程中不一致 体积收缩引起的形状变形的曲率特征。

(2) 结合 FPP 过程中聚合程度沿固化厚度方向 动态分布的特点, 本文提出针对准一维结构的复合 梁屈曲变形模型, 并建立了变形曲率与曝光剂量间 的关系。然后针对准二维平面变形曲率出现分叉点 的问题, 推导出其变形过程中的弹性势能函数, 并 结合有限元分析方法, 以温度场中的热应力代替收 缩应力进行变形结果的仿真计算。
(3) 利用 FPP 过程中不同平面几何形状的变形 特点, 将三维模型的空间曲率信息映射为二维光编 码图像的灰度值, 实现对平面到曲面形状变形的参 数化设计, 并获得了合理的工艺路线, 一些自变形 三维结构的自组装制造也验证了本文提出的工艺方 法的可行性。

\section{参 考 文 献}

[1] GRACIAS D H. Stimuli responsive self-folding using thin polymer films[J]. Current Opinion in Chemical Engineering, 2013, 2(1): 112-119.

[2] STOYCHEV G, ZAKHARCHENKO S, TURCAUD S, et al. Shape-programmed folding of stimuli-responsive polymer bilayers[J]. ACS Nano, 2012, 6(5): 3925-3934.

[3] GAO Wei, WANG Lanlan, WANG Xingzhe, et al. Magnetic driving flowerlike soft platform: Biomimetic 
fabrication and external regulation[J]. ACS Applied Materials \& Interfaces, 2016, 8(22): 14182-14189.

[4] FORTERRE Y, SKOTHEIM J M, DUMAIS J, et al. How the Venus flytrap snaps[J]. Nature, 2005, 433(7024): 421.

[5] ZHAO Qian, QI H J, XIE Tao. Recent progress in shape memory polymer: New behavior, enabling materials, and mechanistic understanding $[\mathrm{J}]$. Progress in Polymer Science, 2015, 49: 79-120.

[6] 于靖军, 谢岩, 裴旭. 负泊松比超材料研究进展 [J]. 机 械工程学报, 2018, 54(13): 1-28.

YU Jingjun, XIE Yan, PEI Xu. Progress in research on negative Poisson's ratio supermaterials[J]. Journal of Mechanical Engineering, 2018，54(13): 1-28.

[7] 祝连庆, 孙广开, 李红, 等. 智能柔性变形机翼技术的 应用与发展 [J]. 机械工程学报, 2018, 54(13): 28-42. ZHU Lianqing, SUN Guangkai, LI Hong, et al. Application and development of intelligent flexible deformation wing technology[J]. Journal of Mechanical Engineering, 2018, 54(13): 28-42.

[8] KLEIN Y, EFRATI E, SHARON E. Shaping of elastic sheets by prescription of non-Euclidean metrics[J]. Science, 2007, 315(5815): 1116-1120

[9] 魏洪秋, 万雪, 刘彦菊, 等. 4D 打印形状记忆聚合物材 料的研究现状与应用前景 $[\mathrm{J}]$. 中国科学: 技术科学, 2018, 48(1): 6-20.

WEI Hongqiu, WAN Xue, LIU Yanju, et al. Research status and application prospect of 4D print shape memory polymer materials[J]. Chinese Science : Technical Science, 2018, 48(1): 6-20.

[10] VAN MANEN T, JANBAZ S, ZADPOOR A A. Programming the shape-shifting of flat soft matter[J]. Materials Today, 2017, 21(2): 144-163.

[11] IONOV L. Biomimetic 3D self-assembling biomicroconstructs by spontaneous deformation of thin polymer films[J]. Journal of Materials Chemistry, 2012, 22(37): 19366-19375.

[12] MIYASHITA S, GUITRON S, YOSHIDA K, et al. Ingestible, controllable, and degradable origami robot for patching stomach wounds[C]/Robotics and Automation (ICRA), 2016 IEEE International Conference on. IEEE, 2016: 909-916.

[13] SCHROEDER T B H, GUHA A, LAMOUREUX A, et al. An electric-eel-inspired soft power source from stacked hydrogels[J]. Nature, 2017, 552(7684): 214.

[14] WAGNER M, CHEN Tian, SHEA K. Large shape transforming 4D auxetic structures[J]. 3D Printing and Additive Manufacturing, 2017, 4(3): 133-142.

[15] HINES L, PETERSEN K, LUM G Z, et al. Soft actuators for small-scale robotics[J]. Advanced Materials, 2017, 29(13): 1603483.

[16] YOON C K, XIAO Rui, PARK J H, et al. Functional stimuli responsive hydrogel devices by self-folding[J]. Smart Materials and Structures，2014，23(9): 094008.

[17] MIYASHITA S, ONAL C D, RUS D. Self-pop-up cylindrical structure by global heating[C]//Intelligent Robots and Systems (IROS), 2013 IEEE/RSJ International Conference on. IEEE，2013： 4065-4071.

[18] ZHANG Qiuting, WOMMER J, O'ROURKE C, et al. Origami and kirigami inspired self-folding for programming three-dimensional shape shifting of polymer sheets with light $[\mathrm{J}]$. Extreme Mechanics Letters, 2017, 11: 111-120.

[19] GLADMAN A S, MATSUMOTO E A, NUZZO R G, et al. Biomimetic 4D printing[J]. Nature Materials, 2016, 15(4): 413.

[20] DING Z, YUAN C, PENG X, et al. Direct 4D printing via active composite materials[J]. Science Advances, 2017, 3(4): e1602890.

[21] KIM J, HANNA J A, BYUN M, et al. Designing responsive buckled surfaces by halftone gel lithography[J]. Science, 2012, 335(6073): 1201-1205.

[22] LI Moxiao, YANG Qingzhen, LIU Hao, et al. Capillary origami inspired fabrication of complex $3 \mathrm{~d}$ hydrogel constructs[J]. Small, 2016, 12(33): 4492-4500.

[23] ZHANG Yihui, ZHANG Fan, YAN Zheng, et al. Printing, folding and assembly methods for forming 3D mesostructures in advanced materials[J]. Nature Reviews Materials, 2017, 2(4): 17019.

[24] GUSEINOV R, MIGUEL E, BICKEL B. CurveUps: Shaping objects from flat plates with tension-actuated curvature[J]. ACM Transactions on Graphics (TOG), 2017, 36(4): 64-76.

[25] ZHAO Zeang, WU Jiangtao, MU Xiaoming, et al. Desolvation induced origami of photocurable polymers by digit light processing[J]. Macromolecular Rapid Communications, 2017, 38(13): 1600625.

[26] HUANG Limei, JIANG Ruiqi, WU Jingjun, et al. Ultrafast digital printing toward 4D shape changing materials[J]. Advanced Materials, 2017, 29(7): 1605390.

[27] ZHAO Zeang, WU Jiangtao, MU Xiaoming, et al. Origami by frontal photopolymerization[J]. Science Advances, 2017, 3(4): e1602326.

[28] GOU Malin, QU Xin, ZHU Wei, et al. Bio-inspired detoxification using 3D-printed hydrogel nanocomposites[J]. Nature communications, 2014, 5: 3774.

(下转第 194 页) 
analysis for helical groove of micro mill based on non-instantaneous-pole envelope principle[J]. Journal of Mechanical Engineering, 2016，52(19): 197-203.

[15] ABELE E, FUJARA M. Simulation-based twist drill design and geometry optimization[J]. CIRP AnnalsManufacturing Technology, 2010, 59(1): 145-150.
作者简介: 梁志强(通信作者), 男, 1984 年出生, 博士, 副教授, 博士 生导师。主要研究方向为难加工材料切削磨削技术, 微细刀具设计与制 造技术。

E-mail: liangzhiqiang@bit.edu.cn

郭海新, 男, 1994 年出生, 硕士研究生。主要研究方向为微细钻削刀具 设计与制造技术。

E-mail: 18811318020@163.com

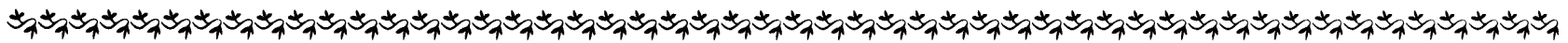

(上接第 184 页)

[29] WU Jiangtao, ZHAO Zeang, HAMEL C M, et al. Evolution of material properties during free radical photopolymerization[J]. Journal of the Mechanics and Physics of Solids, 2018, 112: 25-49.

[30] VITALE A, CABRAL J T. Frontal conversion and uniformity in $3 \mathrm{D}$ printing by photopolymerisation[J]. Materials, 2016, 9(9): 760.

[31] CABRAL J T, DOUGLAS J F. Propagating waves of network formation induced by light $[\mathrm{J}]$. Polymer, 2005, 46(12): 4230-4241.

[32] MU Xiaoming, SOWAN N, TUMBIC J A, et al. Photo-induced bending in a light-activated polymer laminated composite[J]. Soft Matter, 2015, 11(13): 2673-2682.

[33] FREUND L B. Substrate curvature due to thin film mismatch strain in the nonlinear deformation range[J]. Journal of the Mechanics and Physics of Solids, 2000, 48(6-7): 1159-1174.

[34] GE Qi, DUNN C K, QI H J, et al. Active origami by 4D printing[J]. Smart Materials and Structures, 2014, 23(9): 094007.

[35] SHARON E, EFRATI E. The mechanics of non-Euclidean plates[J]. Soft Matter, 2010，6(22): 5693-5704.

[36] SCHNEIDER C A, RASBAND W S, ELICEIRI K W. NIH Image to ImageJ: 25 years of image analysis[J]. Nature Methods，2012，9(7): 671.
[37] GLUGLA D J, ALIM M D, BYARS K D, et al. Rigid origami via optical programming and deferred self-folding of a two-stage photopolymer[J]. ACS Applied Materials \& Interfaces, 2016, 8(43): 29658-29667.

[38] CHESTER S A, DI LEO C V, ANAND L. A finite element implementation of a coupled diffusion-deformation theory for elastomeric gels[J]. International Journal of Solids and Structures, 2015，52: 1-18.

[39] MANSFIELD E H. The bending and stretching of plates[M]. Cambridge: Cambridge University Press, 2005.

[40] ABDULLAH A M, BRAUN P V, HSIA K J. Bifurcation of self-folded polygonal bilayers[J]. Applied Physics Letters, 2017, 111(10): 104101.

[41] ALBEN S, BALAKRISNAN B, SMELA E. Edge effects determine the direction of bilayer bending[J]. Nano Letters, 2011, 11(6): 2280-2285.

[42] ABDULlAH A M, BRAUN P V, HSIA K J. Programmable shape transformation of elastic spherical domes[J]. Soft Matter, 2016, 12(29): 6184-6195.

作者简介: 王金强, 男, 1992 年出生。主要研究方向为数字化设计制造, 自组装结构设计。

E-mail: wangjq@nuaa.edu.cn

戴宁(通信作者), 男, 1978 年出生, 博士, 副教授, 硕士研究生导师。 主要研究方向为增材设计制造技术、生物医学工程、逆向工程技术。 E-mail: dai_ning@nuaa.edu.cn 